\title{
Experimental achievement of the entanglement assisted capacity for the depolarizing channel
}

\author{
Andrea Chiuri ${ }^{1}$, Sandro Giacomini ${ }^{1}$, Chiara Macchiavello ${ }^{2}$, and Paolo Mataloni ${ }^{1,3}$ \\ ${ }^{1}$ Dipartimento di Fisica, Sapienza Università di Roma, Piazzale Aldo Moro 5, I-00185 Roma, Italy \\ ${ }^{2}$ Dipartimento di Fisica and INFN-Sezione di Pavia, via Bassi 6, 27100 Pavia, Italy \\ ${ }^{3}$ Istituto Nazionale di Ottica (INO-CNR), Largo E. Fermi 6, I-50125 Firenze, Italy
}

(Dated: September 8, 2018)

\begin{abstract}
We experimentally demonstrate the achievement of the entanglement assisted capacity for classical information transmission over a depolarizing channel. The implementation is based on the generation and local manipulation of 2-qubit Bell states, which are finally measured at the receiver by a complete Bell state analysis. The depolarizing channel is realized by introducing quantum noise in a controlled way on one of the two qubits. This work demonstrates the achievement of the maximum allowed amount of information that can be shared in the presence of noise and the highest reported value in the noiseless case.
\end{abstract}

PACS numbers: 42.50.Dv,03.67.Bg,42.50.Ex

Introduction.- Noise is unavoidably present in any realistic implementation of a communication channel. It is therefore of great importance to design strategies that allow to optimise the flow of information transmitted in presence of noise. In the most general scenario information can be transmitted by quantum states and several notions of efficiency can be defined according to the considered task and the resource available along the transmission channel. For example, a quantum communication channel can be designed to transmit classical [1], private classical [2] or quantum information [3], and it can be employed on its own or with the addition of other resources, such as entanglement. The case of an entanglement assisted quantum communication channel occurs when classical information is transmitted and entanglement is a priori available between sender and receiver [4]. The capacity is then given by the maximum amount of information that can be transmitted over the channel by optimising the input signals and the output decoding procedure. In this paper we consider the latter scenario and we present an experimental demonstration of the best performance obtained by an entanglement assisted depolarizing channel for qubits, that allows to achieve the information capacity. The experimental realization presented in this work relies on a quantum optical implementation, but it lays the ground for new perspectives of applications to a great variety of communication scenarios. We want to stress that this is the first experimental demonstration of the information capacity for a controlled noisy quantum communication channel, since previous experimental realisations consider just the case of a noiseless channel [5] or classical noise [6].

An entanglement assisted communication scenario is given by a (generally noisy) quantum channel along which quantum states can be transmitted by assuming that an unlimited amount of noiseless entanglement is a priori available between the sender and the receiver [4]. The entanglement assisted classical capacity (EACC) is then given by the maximum amount of classical mutual information that can be transmitted over such a channel. In this work we consider the case of a depolarizing qubit channel [7], where the action on a given quantum state of a two-dimensional system $\rho$ can be described as

$$
\Gamma_{\{p\}}[\rho]=\sum_{i=0}^{3} p_{i} \sigma_{i} \rho \sigma_{i}
$$

Here $\sigma_{0}$ is the identity operator, $\left\{\sigma_{i}\right\}(i=1,2,3)$ are the three Pauli operators $\sigma_{x}, \sigma_{y}, \sigma_{z}$ respectively, and $p_{0}=1-p$ (with $p \in[0,1])$, while $p_{i}=p / 3$ for $i=1,2,3$.

In this case the entanglement assisted classical capacity takes the simple form [4]

$$
C=2+(1-p) \log _{2}(1-p)+p \log _{2}(p / 3) .
$$

The scheme considered in this work is composed of two qubits, initially prepared in the singlet state $\left|\psi^{-}\right\rangle=$ $\frac{1}{\sqrt{2}}(|01\rangle-|10\rangle)$, where the states $|0\rangle$ and $|1\rangle$ are a basis for each qubit. The singlet state then represents the noiseless entangled state that is a priori shared by the sender and the receiver. Classical information is then encoded by the sender by performing, with equal probabilities, either the identity or one of the three Pauli operators on his qubit, which is then transmitted along the depolarizing channel to the receiver. The receiver finally performs a Bell measurement in order to retrieve the information encoded in the two-qubit system. This scenario allows to achieve the capacity [2] [8].

In this work we implement an experimental scheme corresponding to the above scenario and demonstrate that the capacity (2) can actually be achieved, allowing the optimal transmission of classical information through the channel and therefore the use of the channel at the best of its possible performances. We then measure the classical information transmitted through the channel in terms of the classical mutual information [9], which can be expressed as

$$
I=\sum_{x} p_{1}(x) \sum_{y} p(y \mid x) \log _{2} \frac{p(y \mid x)}{p_{2}(y)}
$$

where $x$ and $y$ are the input/output variables, with corresponding probability distributions $p_{1}(x)$ and $p_{2}(y)$, while $p(y \mid x)$ represents the conditional probability of receiving $y$ 


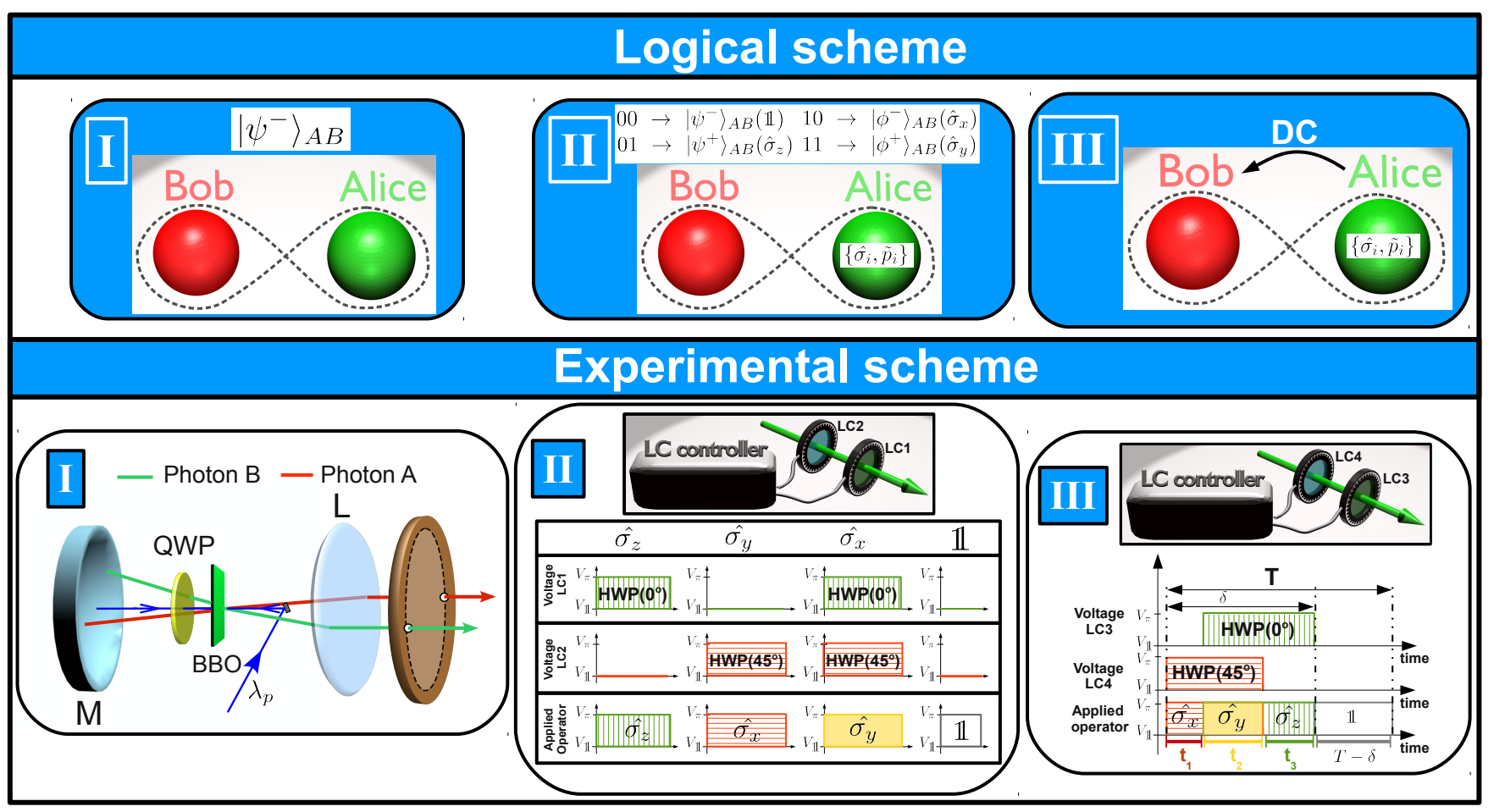

FIG. 1. I) Spontaneous parametric down conversion (SPDC) source of polarization entanglement. A Beta-Barium Borate (BBO) nonlinear crystal is shined by a vertically polarized continuous wave (cw) UV-laser $\left(\lambda_{p}=364 \mathrm{~nm}\right)$, and the two photons are emitted at degenerate wavelength $\lambda=728 \mathrm{~nm}$ with horizontal polarization. Polarization entanglement is generated by the double passage (back and forth, after the reflection on the spherical mirror M) of the UV beam. The backward emission generates the so-called V cone: the SPDC horizontally polarized photons passing twice through the quarter-wave plate (QWP) are transformed into vertically polarized photons. The forward emission generates the $\mathrm{H}$ cone. Because of the temporal and spatial superposition, the indistinguishability of the two perpendicularly polarized SPDC cones creates polarization entanglement $\left(|H\rangle_{A}|H\rangle_{B}+|V\rangle_{A}|V\rangle_{B}\right) / \sqrt{2}$. The relative phase between the states $|H H\rangle_{A B}$ and $|V V\rangle_{A B}$ can be varied by translation of spherical mirror M. A lens $L$ located at a focal distance from the crystal transforms the conical emission into a cylindrical one. II) Alice encodes two bits of classical information by applying single qubit Pauli transformations on one entangled particle. This can be realized by applying a suitable voltage to the liquid crystal modulator, LC1 and LC2 acting as waveplates and implementing the transformations $\left\{\hat{\sigma}_{i}, \tilde{p}_{i}\right\}$. III) Photon A is transmitted through a depolarizing channel (DC), here implemented by liquid crystal modulators LC3 and LC4. The LCs's activation time corresponding to the three Pauli operators $\hat{\sigma}_{x}, \hat{\sigma}_{y}, \hat{\sigma}_{z}$ is the same, i.e. $t_{1}=t_{2}=t_{3}$.

given transmission of $x$. In our experiment the variables $x$ and $y$ correspond to the four Bell states, $p_{1}(x)=1 / 4$ and $p(y \mid x)$ is the conditional probability of detecting the Bell state $y$ given that the Bell state $x$ is transmitted.

General scheme.- We describe here the general experimental scheme to implementing the scenario described above. The two-photon Bell states are engineered by exploiting a polarization entanglement source [10, 11] [See Fig,1])] which allows to generate accurately the states $\left|\phi^{ \pm}\right\rangle=\frac{1}{\sqrt{2}}(|00\rangle \pm|11\rangle)$, with qubit $|0\rangle(|1\rangle)$ corresponding to the horizontal $H$ (vertical $V$ ) polarization of the photon. The other Bell states $\left|\psi^{ \pm}\right\rangle=\frac{1}{\sqrt{2}}(|01\rangle \pm|10\rangle)$ can be obtained by applying simple single-qubit local operations. Let us now assume that the initial state, shared by the sender Alice and the receiver Bob, is represented by the singlet $\left|\psi^{-}\right\rangle_{A B}=\frac{1}{\sqrt{2}}(|01\rangle-|10\rangle)$. In this case Alice encodes classical information by performing local transformations on photon $\mathrm{A}$, which is then transmitted to Bob through the depolarising channel, while photon B belongs to Bob.
In the experiment the information encoding was performed by employing two liquid crystals (LC1 and LC2) acting simultaneously on photon A. This corresponds to apply the single qubit local operation $\hat{\sigma}_{i}$. The LCs acted as phase retarders, with relative phase between the ordinary and extraordinary radiation components depending on the applied voltage $V$. Precisely, $V_{\pi}$ and $V_{\mathbb{1}}$ (Fig]1 I ) corresponded to the case of LCs operating as half-waveplate (HWP) and as the identity operator, respectively. The LC1 and LC2 optical axes were set at $0^{\circ}$ and $45^{\circ}$ with respect to the V-polarization. When the voltage $V_{\pi}$ was applied, LC1 (LC2) acted as a $\sigma_{z}\left(\sigma_{x}\right)$ on the single qubit. The simultaneous application of $V_{\pi}$ on both LC1 and LC2 corresponds to the $\sigma_{y}$ operation. As described in Fig 1II), LCs were suitably activated by a remote control in order to perform the four Pauli operators with equal probabilities. In this way, depending on the transformation implemented by the LCs, Alice encodes two bits of classical information in the shared Bell state, i.e. $\mathbb{1} \rightarrow 00, \hat{\sigma}_{z} \rightarrow 01$, $\hat{\sigma}_{x} \rightarrow 10, \hat{\sigma}_{y} \rightarrow 11$. 


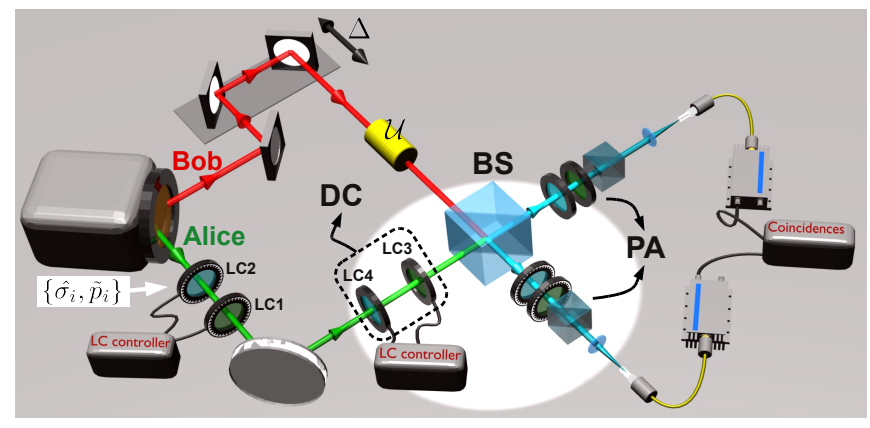

FIG. 2. Experimental setup. Alice and Bob share a two-qubit singlet state. LC1 and LC2 implement the four Pauli operators $\sigma_{i}$ with probability $\tilde{p}_{i}$. The LC3 and LC4 systems perform the DC operation. By properly setting both temporal delay and spatial mode superposition, the two photons arrive in the BS in condition of complete undistinguishability. The noisy state is projected onto the four Bell states by exploiting the $\mathrm{BS}$ and the transformation $\mathcal{U}$. Each polarization analysis (PA) setup consists of a half-waveplate, a quarter-waveplate and a polarizing $\mathrm{BS}$ placed before the detectors.

The manipulated qubit was then sent through a depolarizing channel (DC). Several experimental techniques have been previously proposed and realized to engineer a DC [12-15]. In our case the noisy channel was implemented according to the scheme proposed in [16] [See Fig[1III)]. The necessary logical operations were implemented by careful adjustment of the voltage applied to two LCs and of the activation interval of each LC system. In the next paragraph we will discuss in more detail how the realized noisy channel works.

In order to evaluate the joint probabilities, the four possible outputs $y$ need to be measured for each input state $x$. This final Bell measurement, corresponding to the projective measurement $\left\{\left|\psi^{-}\right\rangle\left\langle\psi^{-}|,| \psi^{+}\right\rangle\left\langle\psi^{+}|,| \phi^{-}\right\rangle\left\langle\phi^{-}|,| \phi^{+}\right\rangle\left\langle\phi^{+}\right|\right\}$, can be realized by using a suitable interferometric setup and by performing those local transformations which allow to select the desired Bell state.

Experimental realization.- Let us consider the expression of the classical mutual information given in Eq. (4). As mentioned above, after encoding, photon $\mathrm{A}$ is transmitted through the depolarizing channel. The experiment was performed by starting from the shared singlet state $\left|\psi^{-}\right\rangle$and for each one of the other Bell states obtained after the encoding operation. The DC was performed by two liquid crystal retarders (LC3 and LC4) inserted within the path of one of the two photons. We were able to switch between $V_{\mathbb{1}}$ and $V_{\pi}$ in a controlled way and independently for both LC3 and LC4. We could also adjust the temporal delay between the intervals corresponding to a $V_{\pi}$ voltage applied to the two retarders. Let us define $t_{1}$, $t_{2}, t_{3}$ respectively as the activation time of the operators $\sigma_{x}$, $\sigma_{y}$ or $\sigma_{z}$ while $T$ is the period of the LCs activation cycle. The condition $t_{1}=t_{2}=t_{3}$ corresponds to the case of a depolarizing channel, with the three Pauli operators acting on the single qubit with the same probability $p_{\exp }=\frac{\delta}{T}=\frac{t_{1}+t_{2}+t_{3}}{T}$. In the experiment, the parameter $p_{\text {exp }}$ was varyed by changing the interval $\delta$ for a fixed period $T$.
We show in Fig. 2 the actual interferometric setup adopted to perform projective measurements $\left\{\left|\psi^{-}\right\rangle\left\langle\psi^{-}|,| \psi^{+}\right\rangle\left\langle\psi^{+}|,| \phi^{-}\right\rangle\left\langle\phi^{-}|,| \phi^{+}\right\rangle\left\langle\phi^{+}\right|\right\}$. The transformation $\mathcal{U}$, sketched in the same figure, represents the different local unitaries necessary to transform each Bell state $\left|\phi^{+}\right\rangle$, $\left|\phi^{-}\right\rangle,\left|\psi^{+}\right\rangle$into the singlet state $\left|\psi^{-}\right\rangle$, i.e. the only one that allows to measure coincidences between the two output modes of the BS in condition of complete undistinguishability. They are implemented by proper setting of the optical axes of a quarter-wave plate and a half-wave plate. In this way we could perform a complete Bell measurement by performing the four projections at different times.

For each value of the noise degree, the probabilities associated to the measurement of each Bell state, corresponding to the conditional probabilities $p(y \mid x)$ of Eq. (4), were obtained by considering the coincidence counts measured by the interferometer for each projection normalized over the results of the four measurements. After the transmission through the beam splitter (BS), photons were coupled into single-mode fibers by using GRaded INdex (GRIN) lenses [17] and detected by single photons detectors.

Experimental results.- Let us consider Eq. (4), with $\mathrm{x}, \mathrm{y}=1, \ldots, 4$ representing the four Bell states. In our case $1 \rightarrow$ $\left|\psi^{-}\right\rangle, 2 \rightarrow\left|\psi^{+}\right\rangle, 3 \rightarrow\left|\phi^{-}\right\rangle, 4 \rightarrow\left|\phi^{+}\right\rangle$. Since the probabilities of the four input Bell states are equal we have $p_{1}(x)=\frac{1}{4}$. Therefore Eq. (4) reads

$$
I_{\text {meas }}=\frac{1}{4} \sum_{x, y} p(y \mid x) \log _{2} \frac{p(y \mid x)}{p_{2}(y)} .
$$

The probabilities $p(y \mid x)$ in the above expression were measured by considering separately each Bell state $x$ and by projecting it onto the four Bell states after the action of the DC. The experiment was carried out for each state obtained after the encoding operation and for several values of the noise degrees, i.e. of the parameter $p_{\text {exp }}$. The values of $I_{\text {meas }}$ were then obtained from the measured probabilities as in Eq. (4).

The experimental data were analyzed by taking into account the non perfect purity of the actual input singlet state. This was evaluated for the singlet state $\left|\psi^{-}\right\rangle$from the visibility $\mathcal{V} \approx 94 \%$ of the coincidence count peak measured in condition of temporal and spatial undistinguishability [see the inset of Fig 3]. According to this value, we could express the actual input state entering the DC as

$$
\rho_{\mathcal{V}}=\mathcal{V}\left|\psi^{-}\right\rangle\left\langle\psi^{-}\right|+(1-\mathcal{V}) \frac{\mathbb{1}}{4}
$$

The above state can be interpreted as the result of the action of a preliminar DC channel on the pure state $\left|\psi^{-}\right\rangle\left\langle\psi^{-}\right|$, characterized by the noise parameter $p^{\prime}=3(1-\mathcal{V}) / 4$. The action of the sequence of two DCs may be then expressed as a global DC with the following global noise parameter

$$
p=\mathcal{V} p_{\exp }+p^{\prime} .
$$


We report in Fig. 3 the experimental results for the transmitted information with the theoretical value of the capacity (2) with $p$ given by Eq. (6). The error bars have been obtained by propagating the poissonian uncertainties associated to the coincidence counts. The agreement between the experimental data analyzed as explained above and the theoretical behaviour is very high.

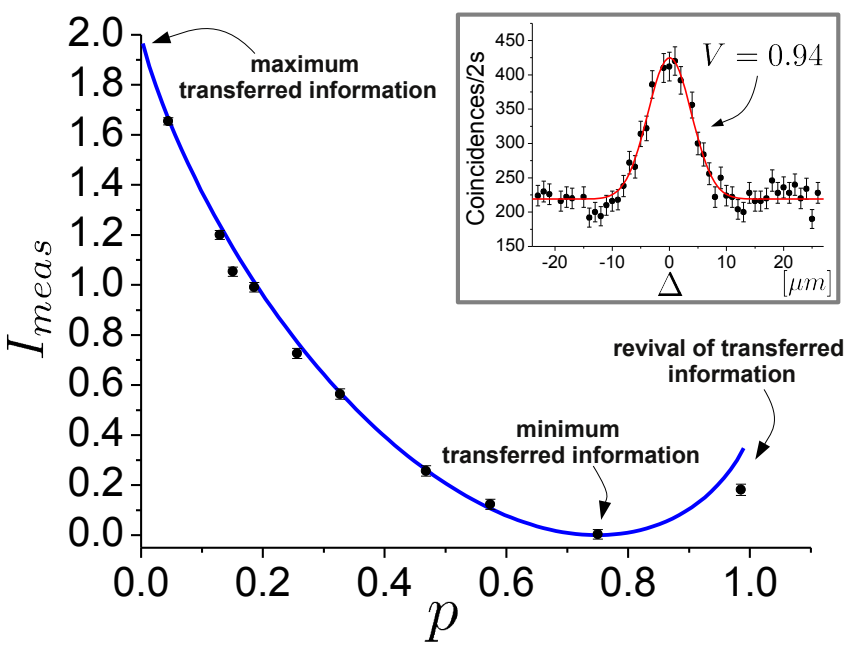

FIG. 3. Measured values (black dots) of the mutual information and theoretical curve (blue line) for the entanglement assisted capacity for several values of the parameter $p$ which fully characterize the total amount of depolarizing noise introduced in the experiment. The error bars have been obtained by propagating the poissonian uncertainties associated to the coincidence counts measured in 10 seconds. Inset: peak of the coincidence counts measured in 2 seconds as a function of the optical path delay $\Delta$ for a state $\left|\psi^{-}\right\rangle\left\langle\psi^{-}\right|$entering the BS in the absence of controlled noise.

Discussions and Conclusions.- The theoretical curve in Fig. 3 allows to single out three working regimes corresponding to three different values of the noise parameter $p$ :

- $p=0$ (absence of noise). The maximum allowed transferred information consists of 2 bits and the noiseless protocol is equivalent to superdense coding [18].

- $p=0.75$. The noisy state is given by the two-qubit maximally mixed state $\frac{\mathbb{1}}{4}$. In these conditions there is no correlation between the output and the input state, hence no information can be transmitted.

- $p>0.75$. In spite of the large amount of noise a partial revival of the mutual information is observable due to the non uniform distribution of the conditional probabilities $p(y \mid x)$.

The capability of exploring the three regimes strongly depends on the purity of the entangled input state. It is remarkable that the purity, attainable from the visibility $\mathcal{V}$ in absence of any active noise applied by LC3 and LC4 (corresponding to an effective noise parameter $p=0.045$ ), easily allows to achieve a quite high value of $I_{\text {meas }}$. Precisely, in this case we obtained $I_{\text {meas }}=1.655 \pm 0.014$ demonstrating a high channel capacity of our system. We want to point out that this value exceeds the threshold for linear-optics implementations reported in [5], where a superdense coding channel capacity equal to 1.630 bits was obtained. Therefore, our result represents also the best performance obtained so far in the framework of superdense coding [19, 20]. As a further consideration, in these experimental conditions, the growing of mutual information occurring for large values of $p(>0.75)$ may be clearly detected.

In this work we have given a proof-of-principle experimental demonstration of the entanglement assisted capacity for classical information transmission over a depolarizing quantum communication channel, where classical information is encoded locally on a preshared maximally entangled state of two qubits and a controlled noise is then introduced on the transmitted qubit. Our experimental implementation of the protocol demonstrates the achievement of the classical information capacity theoretically predicted for the depolarising channel, therefore showing the optimal way in which the depolarising channel can be used when classical information is to be transmitted and a priori entanglement is available.

This work was supported by EU-Project CHISTERAQUASAR, PRIN 2009 and FIRB-Futuro in ricerca HYTEQ.

[1] B. Schumacher and M.D. Westmoreland, Phys. Rev. A 56, 131 (1997); A.S. Holevo, IEEE Trans. Inf. Theory 44, 269 (1998).

[2] I. Devetak, IEEE Trans. Inf. Theory 51, 44 (2005).

[3] S. Lloyd Phys. Rev. A 55, 1613 (1997).

[4] C.H. Bennett et al., Phys. Rev. Lett. 83, 3081 (1999).

[5] See for example the recent implementation by T. Barreiro et al., Nature Physics 4, 282 (2008).

[6] R. Prevedel et al., Phys. Rev. Lett. 106, 110505 (2011).

[7] M. A. Nielsen and I. L. Chuang, Quantum Computation and Quantum Information (Cambridge University Press, Cambridge, England, 2000).

[8] Z. Shadman, H. Kampermann, C. Macchiavello and D. Bruß, New J. Phys. 12, 073042 (2010).

[9] T.M. Cover and J.A. Thomas, Elements of information theory, Wiley \& Sons (1991).

[10] C. Cinelli et al., Phys. Rev. A 70, 022321 (2004).

[11] M. Barbieri et al., Phys. Rev. A 72, 052110 (2005).

[12] M. Ricci et al., Phys. Rev. Lett. 93, 170501 (2004).

[13] M. Karpinski et al., J. Opt. Soc. Am. B 25, 668 (2008).

[14] A. Shaham and H. S. Eisenberg, Phys. Rev. A 83, 022303 (2011).

[15] Y.-C. Jeong et al., arXiv:1204.0850 (2012)

[16] A. Chiuri et al., Phys. Rev. Lett. 107, 253602 (2011).

[17] A. Rossi et al., Phys. Rev. Lett. 102, 153902 (2009).

[18] C. H. Bennett and S. J. Wiesner, Phys. Rev. Lett. 69, 2881 (1992).

[19] K. Mattle, H. Weinfurter, P. G. Kwiat, and A. Zeilinger, Phys. Rev. Lett. 76, 4656 (1996).

[20] Schaetz, T. et al., Phys. Rev. Lett. 93, 040505 (2004). 